\title{
Efficacy and safety of recombinant factor XIII on reducing blood transfusions in cardiac surgery: A randomized, placebo-controlled, multicenter clinical trial
}

\author{
Keyvan Karkouti, MD, ${ }^{\mathrm{a}}$ Christian von Heymann, MD, ${ }^{\mathrm{b}}$ Christian M. Jespersen, MD, ${ }^{\mathrm{c}}$ \\ Wolfgang Korte, MD, ${ }^{\mathrm{d}}$ Jerrold H. Levy, MD, ${ }^{\mathrm{e}}$ Marco Ranucci, MD, ${ }^{\mathrm{f}}$ Frank W. Sellke, MD, ${ }^{\mathrm{g}}$ and \\ Howard K. Song, MD, $\mathrm{PhD}^{\mathrm{h}}$
}

\begin{abstract}
Objectives: Cardiac surgery with cardiopulmonary bypass frequently leads to excessive bleeding, obligating blood product transfusions. Because low factor XIII (FXIII) levels have been associated with bleeding after cardiac surgery, we investigated whether administering recombinant FXIII after cardiopulmonary bypass would reduce transfusions.
\end{abstract}

\begin{abstract}
Methods: In this double-blinded, placebo-controlled, multicenter trial, 409 cardiac surgical patients at moderate risk for transfusion were randomized to receive an intravenous dose of recombinant FXIII, $17.5 \mathrm{IU} / \mathrm{kg}(\mathrm{n}=143)$, $35 \mathrm{IU} / \mathrm{kg}(\mathrm{n}=138)$, or placebo $(\mathrm{n}=128)$ after cardiopulmonary bypass. Transfusion guidelines were standardized. The primary efficacy outcome was avoidance of allogeneic blood products for 7 days postsurgery. Secondary outcomes included amount of blood products transfused and reoperation rate. Serious adverse events were measured for 7 weeks.

Results: Study groups had comparable baseline characteristics and an approximately $40 \%$ decrease in FXIII levels after cardiopulmonary bypass. Thirty minutes postdose, FXIII levels were restored to higher than the lower 2.5th percentile of preoperative activity in $49 \%$ of the placebo group, and $85 \%$ and $95 \%$ of the 17.5 and 35-IU/kg recombinant FXIII groups, respectively $(P<.05$ for both treatments vs placebo). Transfusion avoidance rates were $64.8 \%, 64.3 \%$, and $65.9 \%$ with placebo, $17.5 \mathrm{IU} / \mathrm{kg}$, and $35 \mathrm{IU} / \mathrm{kg}$ recombinant FXIII (respective odds ratios against placebo, 1.05 [95\% confidence interval, 0.61-1.80] and 0.99 [95\% confidence interval, 0.57-1.72]). Groups had comparable adverse event rates.
\end{abstract}

Conclusions: Replenishment of FXIII levels after cardiopulmonary bypass had no effect on transfusion avoidance, transfusion requirements, or reoperation in moderate-risk cardiac surgery patients (ClinicalTrials.gov identifier: NCT00914589). (J Thorac Cardiovasc Surg 2013;146:927-39)

Cardiac surgery requiring the use of cardiopulmonary bypass $(\mathrm{CPB})$ is frequently complicated by coagulopathy. ${ }^{1}$ Timely control of the coagulopathy is essential if patients are to avoid excessive blood loss, red blood cell transfusions, or surgical reexploration, all of which have been

\footnotetext{
From the Department of Anesthesia and the Toronto General Research Institute, ${ }^{\text {a }}$ Toronto General Hospital, University Health Network, University of Toronto, Toronto, Ontario, Canada; the Department of Anesthesiology and Intensive Care Medicine, ${ }^{\mathrm{b}}$ Charité-Universitätsmedizin Berlin, Campus Charité Mitte and Campus VirchowKlinikum, Berlin, Germany; Safety Surveillance, ${ }^{\mathrm{c}}$ Global Safety, Novo Nordisk, Bagsværd, Denmark; the Center for Laboratory Medicine, ${ }^{\mathrm{d}}$ St Gallen, Switzerland; the Department of Anesthesiology, Cardiothoracic Anesthesiology and Critical Care, ${ }^{\mathrm{e}}$ Emory University School of Medicine, Atlanta, Ga; the Department of Cardiothoracic-Vascular Anesthesia and Intensive Care ${ }^{\mathrm{f}}$ Istituto di Ricovero e Cura a Carattere Scientifico Policlinico San Donato, San Donato Milanese (Milan), Italy; the Division of Cardiothoracic Surgery, ${ }^{\mathrm{g}}$ Alpert Medical School of Brown University and Rhode Island Hospital, Providence, RI; and the Division of Cardiothoracic Surgery, ${ }^{\text {h }}$ Oregon Health and Science University, Portland, Ore.

The trial was sponsored by Novo Nordisk A/S (Bagsværd, Denmark). Dr Karkouti is supported in part by a merit award from the Department of Anesthesia, University of Toronto, Toronto, Ontario, Canada.

Disclosures: Keyvan Karkouti has received honoraria from Novo Nordisk and Bayer. Christian von Heymann has, in the past 3 years, received honoraria for consulting or lecturing from Novo Nordisk, CSL Behring, Boehringer Ingelheim, Bayer HealthCare GmbH, Janssen-Cilag Pharma GmbH, Vifor Pharma, and Pfizer $\mathrm{GmbH}$, has served on an advisory board or steering committee for The Medicines
}

linked to serious adverse outcomes and prolonged hospital stay. ${ }^{1-4}$ The causes of coagulopathy after cardiac surgery are multifactorial and include excessive fibrinolysis, platelet dysfunction, and coagulation factor deficiency due to excessive consumption, dilution, or both. ${ }^{1,2}$ The mainstay

Company, Vifor Pharma, Novo Nordisk, Boehringer Ingelheim Pharma GmbH, Bayer HealthCare, Pfizer $\mathrm{GmbH}$, has received research grant funding from Bayer HealthCare $\mathrm{GmbH}$, Baxter Innovations $\mathrm{GmbH}$, Boehringer Ingelheim Pharma $\mathrm{GmbH}$, and Novo Nordisk, and has received institutional support from the Charité-University Medicine Berlin. Christian M. Jespersen is employed by Novo Nordisk and has shares in the company. Wolfgang Korte received honoraria or support from Novo Nordisk, CSL Behring, Bayer HealthCare, Baxter, Pfizer, Boehringer Ingelheim, AstraZeneca, Daichii, Sanofi Aventis, Vifor, Roche Diagnostics, and Siemens. Jerrold Levy has received honoraria from Boehringer Ingelheim and Merck and research support from ViroPharma. Marco Ranucci has received honoraria and research support from Novo Nordisk, CSL Behring, and Grifols, and is currently a consultant for the Sorin Group. The other authors have nothing to disclose with regard to commercial support.

Received for publication Dec 17, 2012; revisions received April 11, 2013; accepted for publication April 25, 2013; available ahead of print July 1, 2013

Address for reprints: Keyvan Karkouti, MD, Department of Anesthesia, Toronto General Hospital, 3EN, Toronto, Ontario M5G 2C4, Canada (E-mail: keyvan. karkouti@uhn.ca).

0022-5223/\$36.00

Copyright $(2013$ by The American Association for Thoracic Surgery

http://dx.doi.org/10.1016/j.jtcvs.2013.04.044 


\section{Abbreviations and Acronyms \\ $\mathrm{AE}=$ adverse event \\ $\mathrm{CPB}=$ cardiopulmonary bypass \\ $\mathrm{FXIII}=$ factor XIII}

of therapy for coagulopathy in the setting of cardiac surgery includes antifibrinolytic drug therapy to prevent excessive fibrinolysis, platelet transfusions to increase the number of functioning platelets, and plasma transfusions to replenish coagulation factor levels. ${ }^{1}$ These therapies, however, are frequently ineffective and are themselves associated with adverse outcomes. ${ }^{5-7}$ Thus, there remains a need for novel therapies that can effectively and safely prevent or treat coagulopathy after cardiac surgery.

Several plasma-derived and recombinant coagulation factors are available for systemic administration, and their hemostatic effectiveness is under clinical investigation. One potential candidate is recombinant factor XIII (FXIII). FXIII is the terminal enzyme in the coagulation cascade, and is necessary for cross-linking of fibrin monomers to form a stable and strong fibrin clot. ${ }^{8-10}$ Cardiac surgery with CPB leads to a marked reduction in plasma FXIII levels, ${ }^{11-16}$ and an association between low FXIII levels and increased bleeding has been noted in cardiac surgery and other clinical settings, ${ }^{12-17}$ but this finding is not consistent. ${ }^{18}$ Recombinant FXIII has already undergone preliminary clinical trials, ${ }^{19,20}$ and in cardiac surgery, it has been well tolerated in doses from 11.9 to $50 \mathrm{IU} / \mathrm{kg}$, with $35 \mathrm{IU} / \mathrm{kg}$ suggested as possibly the most appropriate dose for replenishment of FXIII levels after cardiac surgery with $\mathrm{CPB} .{ }^{20}$ In the current clinical trial, we assessed the hemostatic efficacy of 2 doses of recombinant FXIII $(17.5$ and $35 \mathrm{IU} / \mathrm{kg})$ administered at the conclusion of $\mathrm{CPB}$ in patients who were at moderate risk for requiring perioperative transfusions due to blood loss to determine whether such treatment would reduce transfusion needs.

\section{METHODS}

This was a randomized, double-blinded, placebo-controlled, multinational, multicenter, phase 2, dose-finding trial conducted from July 27 , 2009, to February 23, 2011. The trial was performed in accordance with the Declaration of Helsinki, International Conference on Harmonization Good Clinical Practice, and was approved by the health authorities of each of the countries involved and by the independent ethics committees or institutional review boards of each center involved. All patients provided written informed consent before involvement in the study. Details of the centers are presented in Appendix 1. The trial was registered at http:// www.clinicaltrials.gov (identifier: NCT00914589).

\section{Patients}

Adult patients (aged 18-80 years) who were scheduled to undergo nonemergent coronary artery bypass grafting requiring $\mathrm{CPB}$, single heart valve replacement or repair requiring $\mathrm{CPB}$, or both of these surgical interventions were eligible for inclusion. Intraoperative antifibrinolytic intervention was mandatory, and patients were required to be at moderate risk for transfusion. A moderate risk for transfusion was defined as meeting 2 or 3 of the following criteria: (1) nonelective surgery, (2) redo surgery, (3) combined coronary artery bypass grafting and valve surgery, (4) aged 69 years or older, (5) body surface area lower than $1.9 \mathrm{~m}^{2},(6)$ creatinine level higher than $100 \mu \mathrm{mol} / \mathrm{L}$ if female and $110 \mu \mathrm{mol} / \mathrm{L}$ if male, (7) platelet count lower than $150,000 / \mu \mathrm{L}$, and (8) hematocrit lower than $36 \%$ if female and $39 \%$ if male. ${ }^{21,22}$ The validity of the criteria for identifying patients at moderate risk for transfusion was confirmed at 2 of the participating centers before trial initiation by retrospectively assessing the transfusion rates in patients who would have met the inclusion criteria. FXIII levels were not considered for inclusion of patients into the study.

Patients were excluded at screening if they had any of the following conditions: history of coagulation disorders, autoimmune diseases, thromboembolic events (excluding cardiac), stroke, or ST-elevated myocardial infarction within 7 days of surgery; received adenosine diphosphate receptor antagonists within 3 days of surgery, glycoprotein IIb/IIIa receptor blockers within 24 hours of surgery, low-molecular-weight heparin within 24 hours of surgery, or vitamin K antagonist therapy accompanied by an international normalized ratio higher than 1.5 on the day of surgery; weight of $140 \mathrm{~kg}$ or higher; left ventricular ejection fraction lower than $30 \%$; liver dysfunction (aspartate aminotransferase or alanine aminotransferase level $>2$-fold higher than the upper limit of normal); platelet count lower than $100 \times 10^{9} / \mathrm{L}$; creatinine level of $175 \mu \mathrm{mol} / \mathrm{L}$ or higher or receiving dialysis; and hematocrit predicted to be lower than $27 \%$ during $\mathrm{CPB}$ (based on starting hematocrit and CPB prime volume).

Eligibility for trial drug administration was reevaluated 15 minutes after initiation of protamine dosing for reversal of heparin, and patients were excluded before trial drug administration (but after randomization) if they had received any blood products or fibrin sealants. In addition, patients were excluded if they had a hematocrit lower than $22 \%$ before cross-clamp removal (because they would have a high chance of receiving packed red blood cells irrespective of the amount of post-CPB blood loss) or had experienced any of the following factors that would have changed their transfusion risk from moderate to high: $\mathrm{CPB}$ duration longer than 3 hours, surgery on the aortic arch or descending aorta, hypothermic $\left(<28^{\circ} \mathrm{C}\right)$ circulatory arrest, or severe hemodynamic instability after $\mathrm{CPB}$ that may have necessitated reinstitution of CPB. Patients were also excluded if the trial drug could not be administered within 30 minutes of protamine administration.

\section{Trial Drug Administration}

Eligible patients were randomized (1:1:1) using an interactive voice/ Web response system to a single intravenous dose of 17.5 or $35 \mathrm{IU} / \mathrm{kg}$ (according to lean body mass) of recombinant FXIII or placebo before the induction of anesthesia. Randomization was stratified according to trial site, transfusion risk score ( 2 or 3 ), age group ( $<65$ or $\geq 65$ years), and whether the patient had undergone previous cardiac surgery. The appearance and physical properties of recombinant FXIII and placebo were indistinguishable. Study drug/placebo was administered via a slow intravenous push $(\leq 2 \mathrm{~mL} / \mathrm{min})$ at 15 to 30 minutes after initiation of protamine dosing after CPB but before any other hemostatic therapies. Induction of anesthesia, dosing with heparin and protamine (to induce and subsequently reverse anticoagulation), and administration of antifibrinolytic treatment (tranexamic acid) were performed according to standard clinical practice at each site.

\section{TRANSFUSION MANAGEMENT}

Autologous blood collected before surgery and acute normovolemic hemodilution were not permitted. Any shed blood collected in the operating room could be retransfused, washed or unwashed, depending on institutional practice. Retransfusion of blood collected in mediastinal drains was not permitted. 
Perioperative transfusion practice was standardized. Red blood cell transfusion was mandatory for hemoglobin lower than $6.0 \mathrm{~g} / \mathrm{dL}$, optional for hemoglobin between 6.0 and 8.0 $\mathrm{g} / \mathrm{dL}$, acceptable for hemoglobin between 8.0 and $10.0 \mathrm{~g} / \mathrm{dL}$ (provided there was evidence for end-organ ischemia), and not permitted for higher hemoglobin values. Transfusions were to be administered $1 \mathrm{U}$ at a time, and the hemoglobin had to be rechecked after each unit. Coagulation products were to be administered if, after reversal of protamine, patients bled $2 \mathrm{~mL} / \mathrm{kg}$ or more over 30 minutes or $1.5 \mathrm{~mL} / \mathrm{kg}$ or more per hour over 2 consecutive hours. Fresh-frozen plasma $(10-15 \mathrm{~mL} / \mathrm{kg})$ was to be administered if the international normalized ratio was 1.5 or higher. Platelets ( 1 apheresis unit or 4-6 pooled units) were to be transfused if the platelet count was $100 \times 10^{9} / \mathrm{L}$ or lower. Fibrinogen concentrate $(2 \mathrm{~g})$ or cryoprecipitate $(8-10 \mathrm{U})$ was to be transfused if the fibrinogen concentration was $1.5 \mathrm{~g} / \mathrm{L}$ or lower. An external adjudication committee was established to ensure compliance with the transfusion protocol at each center.

\section{LABORATORY TESTS}

Blood sampling for clinical laboratory tests was performed before surgery, after protamine, but before trial drug administration, and at the following times after trial drug administration: $30 \pm 5$ minutes, $8 \pm 0.5$ hours, $24 \pm$ 2 hours, $48 \pm 6$ hours, $72 \pm 6$ hours, 7 days or discharge, and 5 to 7 weeks. Standard laboratory tests (hematology, coagulation parameters, biochemistry, and urinalysis) were performed by a local laboratory. Specialty laboratory assessments were performed by a central laboratory; these included FXIII activity (FXIII Berichrom activity assay), FXIII B-subunit (enzyme-linked immunosorbent assay), prothrombin fragment $1+2$, troponin $\mathrm{T}$, fibrinogen, fibrin monomers, and recombinant FXIII antibodies (direct FXIII-antibody enzyme-linked immunosorbent assay).

\section{Pharmacodynamic and Pharmacokinetic Outcomes}

Pharmacodynamic and pharmacokinetic outcomes included plasma FXIII activity levels and the restoration of plasma FXIII activity to higher than the lower 2.5 th percentile of the distribution of preoperative activity.

\section{Efficacy Outcomes}

The primary efficacy end point was the percentage of patients avoiding any allogeneic transfusions (red blood cell, fresh-frozen plasma, platelets, cryoprecipitate, or fibrinogen concentrate) up to postoperative day 7 or hospital discharge, whichever came first. Secondary efficacy end points, which were also assessed up to postoperative day 7 or hospital discharge, were the amount (units) of transfused blood products, the percentage of patients avoiding any red blood cell transfusion, and the percentage of patients avoiding massive red blood cell transfusion ( $\geq 5 \mathrm{U})$.
The incidence of reoperation was assessed until the end of the trial.

\section{Safety}

An external, independent safety data monitoring committee (Appendix 2) was established to ensure the safety of patients. All adverse events (AEs), including severity, relation to trial product, and patient outcome, were reported from the time of trial drug administration until postoperative day 7 or hospital discharge. For serious AEs, reporting continued until the follow-up visit at 5 to 7 weeks after trial drug administration. Prespecified critical AEs were thromboembolic events (including acute myocardial infarction [based on enzyme and electrocardiographic criteria, which were evaluated by an independent central laboratory], stroke, or transient ischemic attack, peripheral artery occlusion, deep venous thrombosis, and pulmonary embolism), renal dysfunction (doubling of creatinine or renal replacement therapy), reoperation, and death.

\section{Statistical Analysis}

The sample size of 409 patients (136 per group) was based on the following assumptions: transfusion avoidance rate of $40 \%$ in the placebo arm (based on the validation of the transfusion risk criteria) and $55 \%$ in each of the treatment arms, 70\% power, and $\alpha$ level of .05 ( 2 sided). The null hypothesis was that placebo and recombinant FXIII administration resulted in the same avoidance rate (ie, odds ratio, 1).

Except where noted, all statistical analyses, including covariate selection, were planned a priori. Transfusion avoidance rates were compared between treatment groups using logistic regression analysis, adjusting for the following covariates: trial site, transfusion risk score ( 2 or 3 ), age group ( $<65$ or $\geq 65$ years), previous cardiac surgery, pretrial drug administration fibrinogen level, pretrial drug administration platelet count, urgency of surgery, and treatment arm. In sensitivity analyses, the primary analysis was repeated, adjusting only for statistically significant covariates and without adjusting for any covariates.

A blinded data review showed that the distribution of the amount of transfusions was not consistent with a Poisson distribution (ie, most patients did not receive any transfusions, and a few patients received many units, with 1 receiving $>80 \mathrm{U}$ ). Therefore, the amount of transfusions was compared using a cumulative logit model on $0,1,2,3,4$, and $>4 \mathrm{U}$. This analysis was adjusted for covariates, as for the primary analysis.

The proportion of patients whose plasma FXIII activity level was restored to higher than the lower 2.5th percentile of the distribution of preoperative activity level was also compared between treatment groups using logistic regression analysis, adjusting for transfusion risk score (2 or 3 ), age, pretrial drug administration FXIII plasma activity, 
and treatment. The plasma concentration of FXIII at each time point was compared among treatment groups using analysis of variance, adjusting for these covariates.

The impact of the achieved FXIII activity level on the avoidance of any transfusions was analyzed in a manner analogous to the primary analysis, except that "treatment" was replaced by FXIII plasma activity at $30 \mathrm{~min}, 24 \mathrm{~h}$, and $48 \mathrm{~h}$ in the respective analyses. The interaction effect between predose FXIII activity and treatment on avoidance of allogeneic transfusions was analyzed in a model similar to the primary analysis, but with predose FXIII activity as a covariate both on its own and in interaction with treatment.

The rate of critical AEs was compared between treatment groups using logistic regression analysis, adjusting for transfusion risk score ( 2 or 3 ), age, type of surgery (elective vs nonelective), and treatment.

The trial was sponsored by Novo Nordisk A/S (Bagsværd, Denmark). The authors designed the trial protocol, directed the statistical plan, and wrote the manuscript. The sponsor oversaw the trial operations, audited all data, and conducted the statistical analyses. Dr Karkouti signed the clinical trial report, and the authors had full access to and take full responsibility for the integrity of the data. All authors have read and agree with the manuscript as written.

\section{RESULTS \\ Patient Disposition, Demographics, and Operative Details}

Patient disposition is shown in Figure 1. Of the 479 randomized patients, 409 were eligible to receive study drug and were included in the analyses. Within this group, 128 received placebo, 143 received $17.5 \mathrm{IU} / \mathrm{kg}$ of recombinant FXIII, and 138 received $35 \mathrm{IU} / \mathrm{kg}$ of recombinant FXIII. A total of 356 patients completed the entire trial, including the 5- to 7-week postoperative visit.

The 13 European hospitals randomized $61 \%$ of patients, followed by the 5 Canadian hospitals (28\%), 7 US hospitals (5\%), 4 Japanese hospitals (4\%), and 2 Israeli hospitals $(2 \%)$ (Appendix 1). This variability is, in part, the result of the study being initiated first in Europe, followed by Canada, and then in the other countries. Patients had similar characteristics, baseline laboratory values, and operative

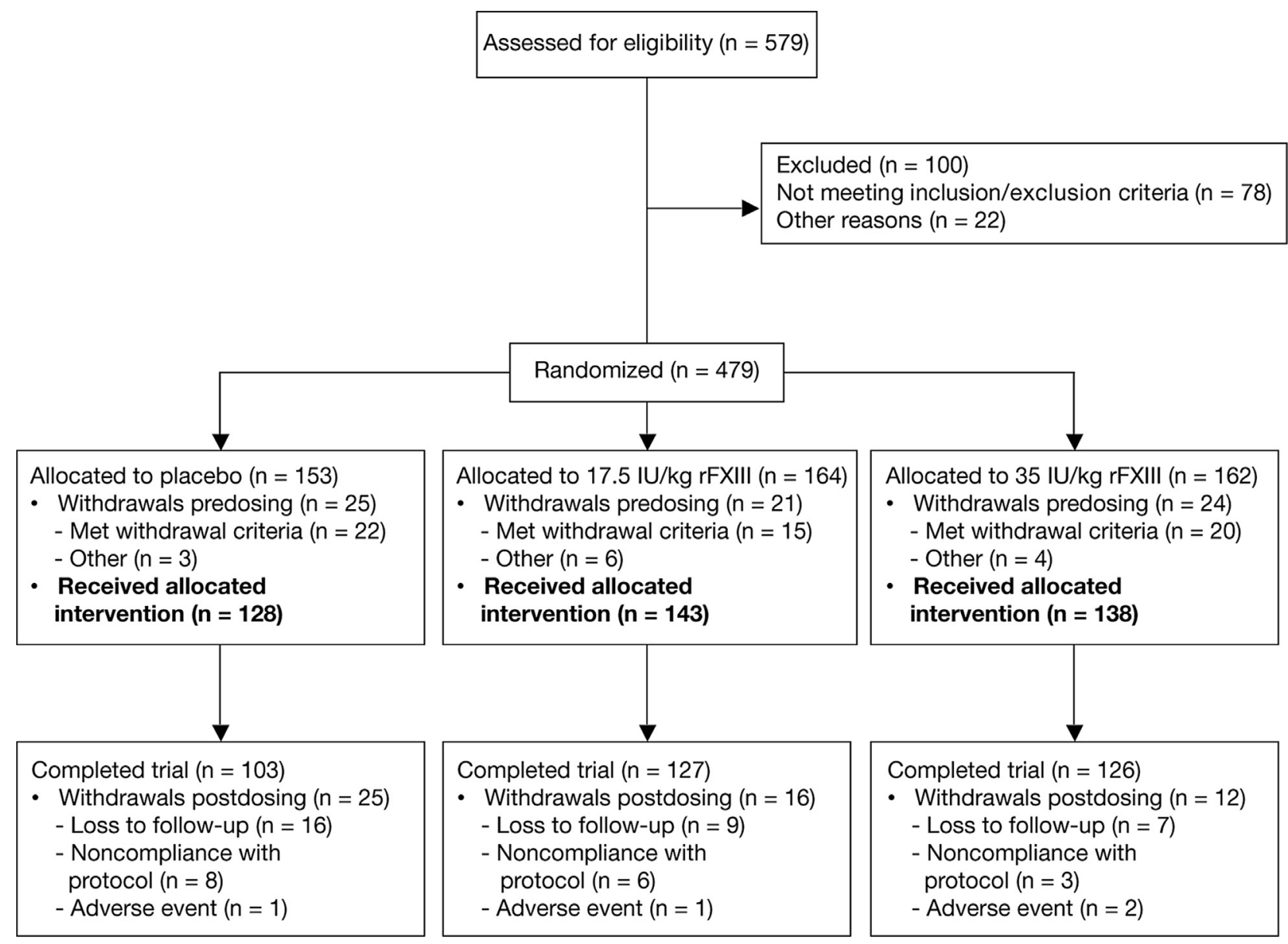

FIGURE 1. Patient disposition. $r F X I I I$, Recombinant factor XIII. 
details across treatment groups (Table 1). All but 8 patients had a transfusion risk score of 2 or 3 .

\section{FXIII Activity Levels}

Baseline FXIII activity levels were similar across treatment groups (Table 1). In addition, all 3 groups had an approximately $40 \%$ decrease in FXIII levels after CPB (Figure 2). Administration of recombinant FXIII increased FXIII levels in a dose-dependent manner, and the levels remained higher than in the placebo group across all 7-day postoperative time points (Figure 2). The proportion of patients who, at 30 minutes postdose, had achieved restoration of FXIII activity to higher than the lower 2.5 th percentile of the preoperative activity was $49 \%$ in the placebo group, $85 \%$ in the $17.5-\mathrm{IU} / \mathrm{kg}$ recombinant FXIII group, and $95 \%$ in the $35-\mathrm{IU} / \mathrm{kg}$ recombinant FXIII group $(P<.05$ in both treatment groups vs placebo).

\section{Efficacy Outcomes}

The administration of recombinant FXIII had no effect on allogeneic transfusion avoidance (Table 2). The proportion of patients who avoided transfusion was $64.8 \%$ with placebo versus $64.3 \%$ with recombinant FXIII, $17.5 \mathrm{IU} / \mathrm{kg}$, and $65.9 \%$ with recombinant FXIII, $35 \mathrm{IU} / \mathrm{kg}$. This translated to an odds ratio close to unity for the comparisons of active treatments against placebo in the applied logistic regression analysis (1.05 [95\% confidence interval, 0.611.80] for the $17.5-\mathrm{IU} / \mathrm{kg}$ recombinant FXIII group and 0.99 [95\% confidence interval, $0.57-1.72]$ for the 35-IU/ $\mathrm{kg}$ recombinant FXIII groups). Although transfusion practice was protocolized and there were few protocol violations $(\mathrm{n}=30)$, transfusion rates varied substantially across sites; the median rate was $29 \%$, and the 25 th and 75 th percentiles were $24 \%$ and $50 \%$ (sites with $\leq 3$ randomized patients were considered a single site for this calculation), with a transfusion rate of $30 \%$. Trial site $(P=.0001)$ was the most important determinant of transfusion avoidance. The results of sensitivity analyses were similar to those of the main analysis.

Subgroup and interaction analyses showed no effect of recombinant FXIII on transfusion avoidance in any of the analyzed subgroups defined by predose FXIII activity, predose fibrinogen concentration, transfusion risk marker, and type of surgery. No interaction between treatment and predose FXIII activity level was identified from logistic regression analysis of transfusion avoidance $(P=.60)$.

TABLE 1. Patient characteristics and operative details

\begin{tabular}{|c|c|c|c|}
\hline & & rFXIII & rFXIII \\
\hline & $\operatorname{Placebo}(n=128)$ & $17.5 \mathrm{IU} / \mathrm{kg}(\mathrm{n}=143)$ & $35 \mathrm{IU} / \mathrm{kg}(\mathrm{n}=\mathbf{1 3 8})$ \\
\hline \multicolumn{4}{|l|}{ Patient characteristics at screening } \\
\hline Age, mean (SD), y & $69(9)$ & $69(9)$ & $69(8)$ \\
\hline Male sex, no. $(\%)$ & $100(78.1)$ & $119(83.2)$ & $117(84.8)$ \\
\hline White race, no. $(\%)$ & $117(91.4)$ & $129(90.2)$ & $125(90.6)$ \\
\hline Body surface area, mean (SD), $\mathrm{m}^{2}$ & $1.9(0.2)$ & $1.9(0.2)$ & $1.9(0.2)$ \\
\hline Left ventricular ejection fraction, mean (SD), $\%$ & $56(10)$ & $57(11)$ & $56(9)$ \\
\hline \multicolumn{4}{|l|}{ Baseline assessments before anesthesia* } \\
\hline Hemoglobin, mean (SD), g/dL & $13.7(1.3)$ & $13.6(1.3)$ & $13.7(1.2)$ \\
\hline Creatinine, mean (SD), $\mu \mathrm{mol} / \mathrm{L}$ & $88(22)$ & $90(23)$ & $93(22)$ \\
\hline Platelet count, mean $(\mathrm{SD}), \times 10^{9} / \mathrm{L}$ & $210(63)$ & $210(61)$ & $212(57)$ \\
\hline INR, mean (SD) & $1.1(0.1)$ & $1.1(0.1)$ & $1.1(0.1)$ \\
\hline Fibrinogen, mean (SD), mg/dL & $406(129)$ & $401(138)$ & $426(148)$ \\
\hline FXIII activity, mean (SD), IU/mL & $1.23(0.26)$ & $1.14(0.28)$ & $1.20(0.29)$ \\
\hline FXIII B-subunit, mean (SD), $\mu \mathrm{g} / \mathrm{mL}$ & $4.71(1.65)$ & $4.91(1.88)$ & $4.80(1.92)$ \\
\hline \multicolumn{4}{|l|}{ Operative details } \\
\hline CABG, no. $(\%)$ & $56(43.8)$ & $79(55.2)$ & $79(57.2)$ \\
\hline Valve repair or replacement, no. $(\%)$ & $39(30.5)$ & $36(25.2)$ & $36(26.1)$ \\
\hline Combined CABG and valve, no. (\%) & $33(25.8)$ & $28(19.6)$ & $23(16.7)$ \\
\hline Redo surgery, no. $(\%)$ & $6(4.7)$ & $3(2.1)$ & $11(8.0)$ \\
\hline Nonelective surgery, no. $(\%)$ & $33(25.8)$ & $44(30.8)$ & $44(31.9)$ \\
\hline EuroSCORE, mean (SD) & $4.3(2.1)$ & $4.1(2.0)$ & $4.3(2.1)$ \\
\hline \multicolumn{4}{|l|}{ No. of transfusion risk criteria, no. $(\%) \dagger$} \\
\hline 1 & $1(0.8)$ & $2(1.4)$ & $1(0.7)$ \\
\hline 2 & $77(60)$ & $82(57)$ & $88(64)$ \\
\hline 3 & $49(38)$ & $57(40)$ & $48(35)$ \\
\hline 4 & $1(0.8)$ & $2(1.4)$ & $1(0.7)$ \\
\hline
\end{tabular}

$r F X I I I$, Recombinant factor XIII; INR, international normalized ratio; $C A B G$, coronary artery bypass grafting. *Baseline values were collected before induction of anesthesia (preinduction). If data were not available, data from the screening visit were used. †See Methods for list of risk factors. 


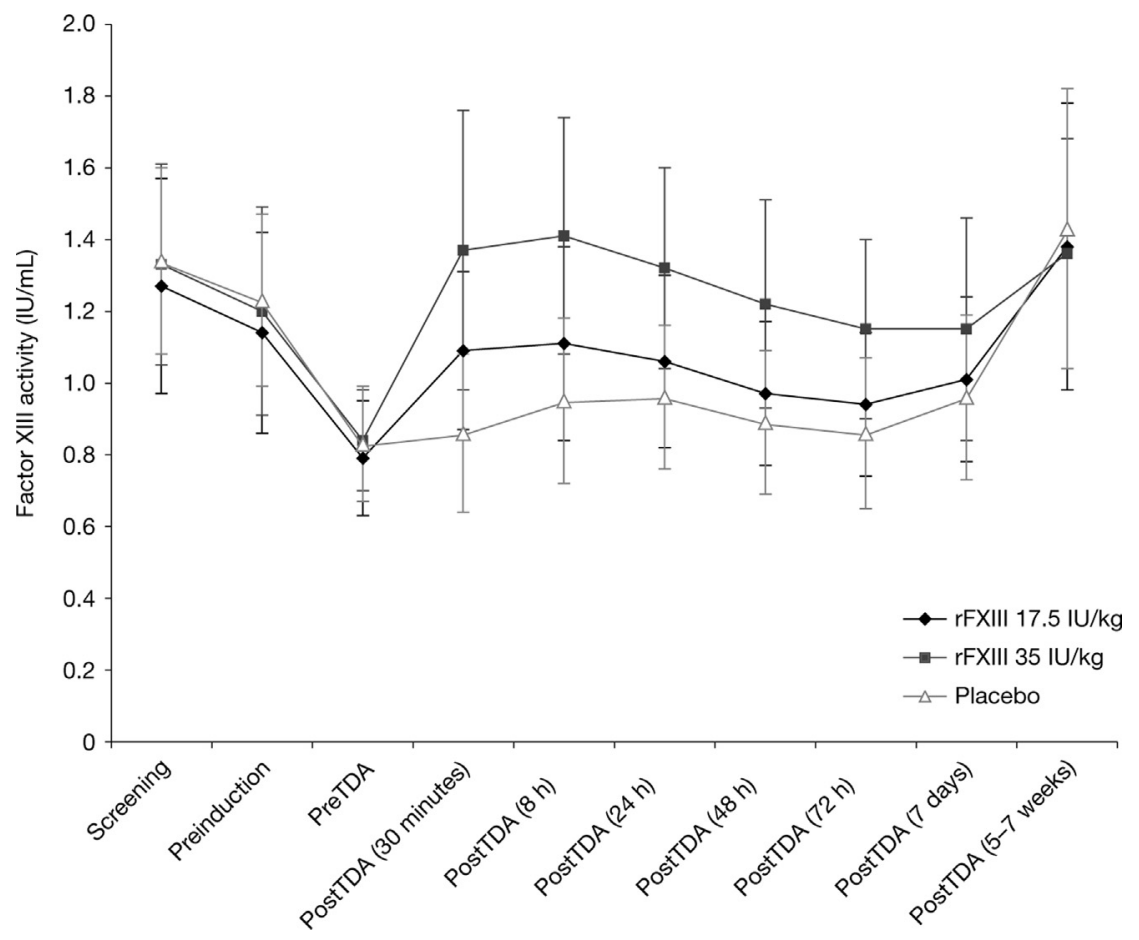

FIGURE 2. Factor XIII levels. Data shown are means and SDs. $r F X I I I$, Recombinant factor XIII; TDA, trial drug administration.

Furthermore, there was no impact of FXIII activity level at 30 minutes postdose on avoidance of allogeneic transfusion $(P=.64)$.

For the secondary transfusion end points, administration of recombinant FXIII did not reduce transfusion requirements, massive red blood cell transfusions, or the incidence of reoperation.

\section{Safety}

Administration of recombinant FXIII was not associated with an increased rate of AEs versus placebo, including all AEs, serious AEs, AEs possibly or probably related to study medication, and withdrawals due to AEs (Table 3). The most commonly reported AEs were pleural effusion $(37 \%)$, atrial fibrillation $(35 \%)$, procedural pain $(30 \%)$, nausea $(29 \%)$, anemia (17\%), peripheral edema (16\%), and hypotension $(16 \%)$. Most AEs were mild or moderate in severity. More important, thromboembolic and predefined critical AEs occurred with similar frequencies in the FXIII and placebo groups. There were 2 fatal AEs (myocardial infarction and sepsis), which both occurred during FXIII treatment but were considered unlikely to be related to trial drug. None of the patients developed antibodies to recombinant FXIII.

\section{DISCUSSION}

In this randomized, double-blinded, placebo-controlled, multicenter clinical trial, we measured the hemostatic efficacy of 17.5 and 35 IU/kg of recombinant FXIII administered at the conclusion of CPB to patients who were at moderate risk for requiring perioperative transfusions because of blood loss. We found that FXIII levels were reduced by approximately $40 \%$ after CPB. We also found that, although both doses of recombinant FXIII

TABLE 2. Efficacy outcomes*

\begin{tabular}{|c|c|c|c|}
\hline & & rFXIII & rFXIII \\
\hline & Placebo $(\mathbf{n}=128)$ & $17.5 \mathrm{IU} / \mathrm{kg}(\mathrm{n}=143)$ & $35 \mathrm{IU} / \mathrm{kg}(\mathrm{n}=138$ \\
\hline \multicolumn{4}{|l|}{ Patients who avoided allogeneic transfusions, no. $(\%)$} \\
\hline All transfusions & $83(64.8)$ & $92(64.3)$ & $91(65.9)$ \\
\hline Red blood cell transfusions & $85(66.4)$ & $94(65.7)$ & $92(66.7)$ \\
\hline Massive ( $\geq 5 \mathrm{U}$ ) red blood cell transfusion & $125(97.7)$ & $140(97.9)$ & $136(98.6)$ \\
\hline Units of transfused blood products, median ( 25 th, 75 th percentiles) & $0(0,1)$ & $0(0,1)$ & $0(0,1)$ \\
\hline Incidence of reoperation, no. $(\%)$ & $2(1.6)$ & $8(5.6)$ & $4(2.9)$ \\
\hline
\end{tabular}

$r F X I I I$, Recombinant factor XIII. *None of the prespecified comparisons was statistically significant. 


\begin{tabular}{|c|c|c|c|}
\hline & & rFXIII & rFXIII \\
\hline & Placebo $(n=128)$ & $17.5 \mathrm{IU} / \mathrm{kg}(\mathrm{n}=143)$ & $35 \mathrm{IU} / \mathrm{kg}(\mathrm{n}=\mathbf{1 3 8})$ \\
\hline All AEs & $126(98.4)$ & $139(97.2)$ & $135(97.8)$ \\
\hline Serious AEs & $35(27.3)$ & $43(30.1)$ & $32(23.2)$ \\
\hline \multicolumn{4}{|l|}{ AEs by severity } \\
\hline Severe & $24(18.8)$ & $31(21.7)$ & $23(16.7)$ \\
\hline Moderate & $91(71.1)$ & $93(65.0)$ & $77(55.8)$ \\
\hline Mild & $111(86.7)$ & $120(83.9)$ & $119(86.2)$ \\
\hline \multicolumn{4}{|l|}{ AEs probably/possibly related to study drug } \\
\hline All & $14(10.9)$ & $17(11.9)$ & $15(10.9)$ \\
\hline Serious AEs & $5(3.9)$ & $6(4.2)$ & $4(2.9)$ \\
\hline Withdrawals due to AEs & $1(0.8)$ & $1(0.7)$ & $1(0.7)$ \\
\hline Thromboembolic events & $12(9.4)$ & $12(8.4)$ & $9(6.5)$ \\
\hline Perioperative acute myocardial infarction & $8(6.3)$ & $10(7.0)$ & $7(5.1)$ \\
\hline Cerebrovascular thromboembolic event & $3(2.3)$ & $2(1.4)$ & 0 \\
\hline Deep vein thrombosis & $1(0.8)$ & 0 & $1(0.7)$ \\
\hline Peripheral artery occlusion & 0 & 0 & $1(0.7)$ \\
\hline Pulmonary embolism & 0 & 0 & 0 \\
\hline Prespecified critical AEs & $19(14.8)$ & $24(16.8)$ & $16(11.6)$ \\
\hline Thromboembolic events & $12(9.4)$ & $12(8.4)$ & $9(6.5)$ \\
\hline Renal dysfunction & $9(7.0)$ & $10(7.0)$ & $5(3.6)$ \\
\hline Reoperation & $2(1.6)$ & $8(5.6)$ & $4(2.9)$ \\
\hline Death & 0 & $1(0.7)$ & $1(0.7)$ \\
\hline
\end{tabular}

Results are reported as number ( $\%)$, where number indicates patients with an AE. $r F X I I I$, Recombinant factor XIII; $A E$, adverse event. *None of the prespecified comparisons was statistically significant.

significantly increased post-CPB FXIII levels, they had no effect on any of the efficacy end points, in either the entire sample or the subset of patients who had low predose FXIII activity. No safety issues were identified, because AEs were evenly distributed across treatment groups and individual types of AEs, including thromboembolic AEs and events predefined as critical for the trial, did not occur with higher frequency in the active dose groups relative to placebo.

The finding that FXIII levels are substantially reduced after CPB is consistent with those of previous studies, which have generally found there is approximately a $40 \%$ decrease in FXIII levels from baseline to after CPB. ${ }^{11-16}$ Although other coagulation factor levels decrease by similar amounts, our hypothesis was based on several lines of evidence that suggest that FXIII substitution after CPB may reduce blood loss. First, several observational studies have found an inverse correlation between FXIII levels after CPB and clot strength or blood loss, ${ }^{12-16}$ but this finding is not consistent. ${ }^{18}$ Second, soluble fibrin monomer levels are increased after $\mathrm{CPB}$, and this increase is independently associated with increased blood loss. ${ }^{11}$ In the presence of adequate FXIII levels, soluble fibrin monomers are cross-linked into stable, insoluble fibrin clots. ${ }^{23}$ Thus, the observed accumulation of soluble fibrin monomers after CPB suggests that there is a relative deficiency of FXIII levels that may contribute to inadequate clot formation and increased bleeding. Third, preliminary studies have shown that FXIII substitution is associated with improved clot strength and reduced blood loss. ${ }^{12,14,24}$

Nevertheless, this study's finding suggests that, at least in patients undergoing moderate-risk cardiac surgery and receiving concomitant antifibrinolytic drug therapy, replenishment of FXIII levels at the conclusion of CPB with recombinant FXIII does not reduce transfusion rates. To our knowledge, 2 other studies have explored the efficacy of FXIII replacement in cardiac surgery. ${ }^{12,14}$ One study included 22 consecutive patients who underwent low-risk cardiac surgery and were alternately assigned to control or FXIII arms (who received $2500 \mathrm{U}$ of FXIII postoperatively). ${ }^{12}$ In that study, patients who received FXIII had lower blood loss and transfusion rates, but only the former achieved statistical significance, and patients were not properly randomized. ${ }^{12}$ More recently, the same group conducted another trial that included 75 patients who underwent low-risk cardiac surgery and were randomized to placebo, low-dose (1250 U), or high-dose (2500 U) FXIII postoperatively (25 patients in each group). ${ }^{14}$ In this blinded study, the amounts of blood loss and transfusion were highest in the placebo group and lowest in the high-dose group, but none of the between-group differences achieved statistical significance. ${ }^{14}$ In both of these studies, patients received concomitant antifibrinolytic therapy (aprotinin), but the transfusion protocol was not standardized.

Several characteristics of this study must be considered when interpreting its negative results. First, based on the 
requirements of some drug-regulating agencies, it was deemed necessary to determine if FXIII replenishment resulted in increased transfusion avoidance. As a result, the sample was limited to patients in whom there was a reasonable chance of transfusion avoidance, which (based on our inclusion criteria) placed them at moderate risk for transfusion. Moreover, despite validating the inclusion criteria at 2 of the participating centers, the transfusion avoidance rate was higher than anticipated, indicating that the transfusion risk in the sample was lower than anticipated. Second, the decision was made to determine the efficacy of FXIII when it is administered early after CPB, irrespective of FXIII levels. Third, the drug was tested in the context of concomitant antifibrinolytic therapy. Thus, it is possible that the drug may have better efficacy in patients whose transfusion risk is higher, in those with severe $(<20 \%$ $30 \%$ activity levels) FXIII deficiency after CPB, or in place of antifibrinolytic drugs. Because FXIII is the terminal enzyme in the coagulation cascade, ${ }^{8-10}$ targeting coagulation factors involved in the earlier stages of coagulation, such as fibrinogen, may be more effective. ${ }^{25}$ Adequately powered randomized controlled trials are needed to examine these issues.

Although this study had many strengths (large, multicenter, placebo-controlled, blinded, randomized trial), it had 2 important weaknesses. First, because this was a phase 2 study, it was not adequately powered to prove drug safety and was only $70 \%$ powered for efficacy. Nevertheless, the efficacy results were clear and there were no major safety concerns detected. Second, despite attempts to standardize transfusion therapy, there were significant variations in transfusion rates across the sites. The reasons for these variations are unclear, but because randomization was stratified according to the site, it is unlikely that this limitation led to unmeasured biases.

In summary, in this multicenter, double-blinded, placebocontrolled, randomized trial, we found that administration of recombinant FXIII (17.5 or $35 \mathrm{IU} / \mathrm{kg}$ ) after cardiac surgery and CPB in patients at moderate risk for transfusion restored FXIII activity to preoperative levels but had no hemostatic benefits, as measured by transfusion avoidance, transfusion requirements, or need for reoperation.

Editorial assistance to the authors during the preparation of the manuscript was provided by Anne Stirland, PhD (medical writer, PAREXEL).

\section{References}

1. Despotis G, Eby C, Lublin DM. A review of transfusion risks and optimal management of perioperative bleeding with cardiac surgery. Transfusion. 2008;48: 2S-30S.

2. Paparella D, Brister SJ, Buchanan MR. Coagulation disorders of cardiopulmonary bypass: a review. Intensive Care Med. 2004;30:1873-81.
3. Christensen MC, Krapf S, Kempel A, von Heymann C. Costs of excessive postoperative hemorrhage in cardiac surgery. J Thorac Cardiovasc Surg. 2009;138: 687-93.

4. Karkouti K, Wijeysundera DN, Yau TM, Beattie WS, Abdelnaem E, McCluskey SA, et al. The independent association of massive blood loss with mortality in cardiac surgery. Transfusion. 2004;44:1453-62.

5. Ferraris VA, Brown JR, Despotis GJ, Hammon JW, Reece TB, Saha SP, et al. 2011 Update to the Society of Thoracic Surgeons and the Society of Cardiovascular Anesthesiologists blood conservation clinical practice guidelines. Ann Thorac Surg. 2011;91:944-82.

6. Bilgin YM, van de Watering LM, Versteegh MI, van Oers MH, Vamvakas EC, Brand A. Postoperative complications associated with transfusion of platelets and plasma in cardiac surgery. Transfusion. 2011;51:2603-10.

7. Alfirevic A, Xu M, Johnston D, Figueroa P, Koch CG. Transfusion increases the risk for vasoplegia after cardiac operations. Ann Thorac Surg. 2011;92:812-9.

8. Ariens RA, Lai TS, Weisel JW, Greenberg CS, Grant PJ. Role of factor XIII in fibrin clot formation and effects of genetic polymorphisms. Blood. 2002;100:743-54.

9. Muszbek L, Bagoly Z, Bereczky Z, Katona E. The involvement of blood coagulation factor XIII in fibrinolysis and thrombosis. Cardiovasc Hematol Agents Med Chem. 2008;6:190-205.

10. Muszbek L, Bagoly Z, Cairo A, Peyvandi F. Novel aspects of factor XIII deficiency. Curr Opin Hematol. 2011;18:366-72.

11. Karkouti K, McCluskey SA, Syed S, Pazaratz C, Poonawala H, Crowther MA. The influence of perioperative coagulation status on postoperative blood loss in complex cardiac surgery: a prospective observational study. Anesth Analg. 2010;110:1533-40.

12. Godje O, Haushofer M, Lamm P, Reichart B. The effect of factor XIII on bleeding in coronary surgery. Thorac Cardiovasc Surg. 1998;46:263-7.

13. Chandler WL, Patel MA, Gravelle L, Soltow LO, Lewis K, Bishop PD, et al. Factor XIIIA and clot strength after cardiopulmonary bypass. Blood Coagul Fibrinolysis. 2001;12:101-8.

14. Godje O, Gallmeier U, Schelian M, Grunewald M, Mair H. Coagulation factor XIII reduces postoperative bleeding after coronary surgery with extracorporeal circulation. Thorac Cardiovasc Surg. 2006;54:26-33.

15. Ternstrom L, Radulovic V, Karlsson M, Baghaei F, Hyllner M, Bylock A, et al. Plasma activity of individual coagulation factors, hemodilution and blood loss after cardiac surgery: a prospective observational study. Thromb Res. 2010;126: e128-33.

16. Shainoff JR, Estafanous FG, Yared JP, DiBello PM, Kottke-Marchant K, Loop FD. Low factor XIIIA levels are associated with increased blood loss after coronary artery bypass grafting. J Thorac Cardiovasc Surg. 1994;108:437-45.

17. Gerlach R, Tolle F, Raabe A, Zimmermann M, Siegemund A, Seifert V. Increased risk for postoperative hemorrhage after intracranial surgery in patients with decreased factor XIII activity: implications of a prospective study. Stroke. 2002; $33: 1618-23$.

18. Blome M, Isgro F, Kiessling AH, Skuras J, Haubelt H, Hellstern P, et al. Relationship between factor XIII activity, fibrinogen, haemostasis screening tests and postoperative bleeding in cardiopulmonary bypass surgery. Thromb Haemost. 2005;93:1101-7.

19. Lovejoy AE, Reynolds TC, Visich JE, Butine MD, Young G, Belvedere MA, et al. Safety and pharmacokinetics of recombinant factor XIII-A2 administration in patients with congenital factor XIII deficiency. Blood. 2006;108:57-62.

20. Levy JH, Gill R, Nussmeier NA, Olsen PS, Andersen HF, Booth FV, et al. Repletion of factor XIII following cardiopulmonary bypass using a recombinant A-subunit homodimer: a preliminary report. Thromb Haemost. 2009;102:765-71.

21. Karkouti K, O'Farrell R, Yau TM, Beattie WS, RBC Reseach Group. Prediction of massive blood transfusion in cardiac surgery. Can J Anesth. 2006;53:781-94.

22. Alghamdi AA, Davis A, Brister S, Corey P, Logan A. Development and validation of Transfusion Risk Understanding Scoring Tool (TRUST) to stratify cardiac surgery patients according to their blood transfusion needs. Transfusion. 2006; 46:1120-9.

23. Spahn DR, Asmis LM. Excessive perioperative bleeding: are fibrin monomers and factor XIII the missing link? Anesthesiology. 2009;110:212-3.

24. Korte WC, Szadkowski C, Gahler A, Gabi K, Kownacki E, Eder M, et al. Factor XIII substitution in surgical cancer patients at high risk for intraoperative bleeding. Anesthesiology. 2009;110:239-45.

25. Sniecinski RM, Levy JH. Bleeding and management of coagulopathy. J Thorac Cardiovasc Surg. 2011;142:662-7. 
APPENDIX TABLE 1. Study sites

Site and principal investigator

Dr Jean-Yves Dupuis

University of Ottawa Heart Institute

40 Ruskin St

Room H545A

Ottawa, ON K1Y 4W7

Canada

Dr Keyvan Karkouti

Toronto General Hospital

200 Elizabeth $\mathrm{St}$

3EN-402

Toronto, ON M5G 2C4

Canada

Dr Louis Perrault

Institut de Cardiologie de Montreal

5000 E Belanger

Department of Anesthesia

Montreal, QC H1T 1C8

Canada

Dr Pierre Voisine

Hospital Laval

2725 Chemin Sainte-Foy

Institut Universitaire de Cardiologie et de

Pneumologie de Québec

Québec, QC G1V 4G5

Canada

Dr C. David Mazer

Saint Michael's Medical Center

30 Bond $\mathrm{St}$

Department of Anesthesia

Toronto, ON M5B-1W8

Canada

Prof Dr Christian von Heymann

Charité-Universitätsmedizin Berlin

Campus Charité Mitte/Campus Virchow-Klinikum

Klinik für Anästhesiologie mit Schwerpunkt

Operative Intensivmedizin

Charitéplatz 1

10117 Berlin

Germany

PD Dr Med Frank Isgro

Klinkum der Stadt Ludwigshafen am Rhein gGmbH

Klinik für Herzchirurgie

Bremserstrasse 79

67063 Ludwigshafen

Germany
Subinvestigators

No. of patients randomized

Dr James Robblee

7

Dr Michael Bourke

Dr Bernard McDonald

Dr Terrence Yau

Dr Stuart McCluskey

Dr Kathleen Dattilo

Dr George Djaiani

Dr Jacek Karski

Dr Michel Pellerin

Dr Michel Carrier

Dr Philippe Demers

Dr Yves Hébert

Dr Denis Bouchard

Dr Antoine Rochon

Dr Raymond Cartier

Dr Richard Baillot

Dr Eric Charbonneau

Dr François Dagenais

Dr Eric Dumont

Dr Daniel Doyle

Dr Patrick Mathieu

Dr Jacques Métras

Dr Jean Perron

Dr Jean Bussières

Dr Marcel Gilbert

Dr Siamak Mohammadi

Dr Mark Peterson

62

Dr David Latter

Dr Daniel Bonneau

Dr Gregory Hare

Prof Dr Michael Sander

Dr Marit Habicher

Dr Torsten Geyer

Dr Juliane Rau

Dr Katharina Berger

Dr Sibylle Semmler

Dr Stephanie Scholz

Dr Med. Birgit Puhlmann

Dr Susanne Niederberger

Dr Med Monica-Cristina Weber 31

Dr Med Michael Neher

Dr Helena Leitao Graca Jourdan

Dr Med Angela Kornberger 
Dr Med Hendrik Ruge

Deutsches Herzzentrum München

Klinik für Hertz-und Gefäßchirurgie

Lazarettstrasse 36

80636München

Germany

Dr Med Arndt-Holger Kiessling

Universitätsklinikum Frankfurt am Main

Theodor Stern Kai 7

60590 Frankfurt am Main

Germany

Dr Peter Skov Olsen

Department of Cardiothoracic Surgery

Copenhagen University Hospital

Blegdamsvej 9, 2100 Copenhagen

Denmark

Dr Alain Vuylsteke

Papworth Hospital NHS Trust

Cambridge, CB3 8RE

UK

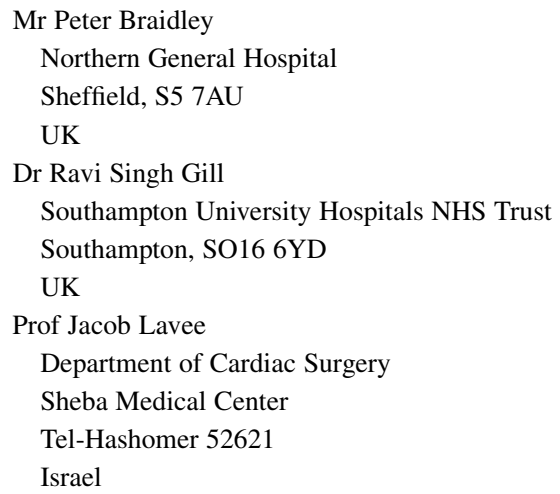

Dr Jacqueline Gümmer

Dr Med Andrea Münsterer

Dr Med Patrick Mayr

Dr Nadejda Monsefi

Prof Daniel Steinbrüchel

Dr Rhiannon Beaumont

Dr Peter Faber

Dr Andrew Klein

Dr Choo Yen Ng

Dr Kamen Valchanov

Dr Stephen Webb

Dr Francis Wells

Dr Tharuka Wijesuriya

Dr Patrick Knowles

Dr Charles Deakin

Dr Michael Herbertson

Dr Paul Diprose

Dr Ehud Raanani

Dr Erez Kachel

Dr Alexander Kogan

Dr Dan Loberman

Dr Vera Coman

Dr Sergey Preisman

Dr Danny Spiegelstein

Dr Leonid Sternik

Dr Basheer Sheick-Yousif

Dr Ami Shinfeld

Dr Yigal Kassif

Dr Prof Aida Inbal

Dr Alexander Lipey

Dr Ehud Jacobzon

Dr Benjamin Medalion

Dr Ariel Farkash

Dr Avi Fuks

Dr Michael Fainblut

Dr Philippe Biderman

Dr Sammer Diab

Dr Eitan Snir

Dr Ram Sharoni

Dr Erez Sharoni

Dr Viacheslav Bobovnikov
9

7

4

4 
Site and principal investigator

Dr Marco Ranucci

Department of Cardiothoracic Anaesthesia and Intensive Care

Istituto di Ricovero e Cura a Carattere Scientifico (IRCCS) Policlinico San Donato

Via R. Morandi 30

20097 San Donato Milanese

Italy

Dr Giovanni Marino

Unità Operativa di Anestesia e Rianimazione

Cardiochirurgica

IRCCS Fondazione Centro S. Raffaele del Monte Tabor

Via Olgettina, 60

20132 Milano

Italy

Dr Atsushi Amano

Department of Cardiovascular Surgery

Tokyo

Japan

Dr Junjiro Kobayashi

Department of Cardiovascular Surgery

National Cardiovascular Center

Osaka

Japan

Dr Hitoshi Okabayashi

Department of Cardiovascular Surgery

Memorial Heart Center

19-1 Uchimaru, Morioka, Iwate 020-8505

Japan

Dr Tatsuhiko Komiya

Department of Cardiovascular Surgery

1-1-1 Miwa, Kurashiki-shi, Okayama, 710-8602

Japan
Subinvestigators

No. of patients randomized

Dr Dario Melani

57

Dr Walter Castracane

Dr Donatella De Benedetti

Dr Angela Satriano

Dr Annalisa Parisi

Dr Elena Bignami

Dr Giuseppe Iaci

Dr Giovanni Landoni

Dr Anna Mizzi

Dr Fabrizio Monaco

Dr Paolo Prati

Dr Leda Nobile

Dr Massimiliano Nuzzi

Dr Alessandro Oriani

Dr Toshimasa Akazawa

1

Dr Shizuyuki Dohi

Dr Naoki Fukuhara

Dr Hirotaka Inaba

Dr Eiichi Inada

Dr Hitomi Iwata

Dr Shiori Kawasaki

Dr Keita Kikuchi

Dr Osamu Kudo

Dr Kenji Kuwaki

Dr Takeshi Matsumura

Dr Taira Yamamoto

Dr Hironobu Yamaoka

Dr Tomoyuki Fujita

Dr Masataka Kamei

Dr Shigeki Miyata

Dr Hiroyuki Nakajima

Dr Tatsuya Ogawa

Dr Yoshihiko Ohnishi

Dr Yusuke Shimahara

Dr Koichi Toda

Dr Kenji Yoshitani

Dr Mamoru Kadosaki

Dr Takeshi Kamada

Dr Kazuyoshi Kanno

Dr Tsuyoshi Kataoka

Dr Hajime Kin

Dr Takashi Kobyashi

Dr Junichi Koizumi

Dr Yoshino Mitsunaga

Dr Masayuki Mukaida

Dr Hiroshi Sato

Dr Junichi Tsuboi

Dr Jouji Ito

Dr Hideyuki Katayama

Dr Norio Mouri

Dr Genichi Sakaguchi

Dr Jiro Sakai 


\begin{tabular}{l}
\hline \\
Francisco Javier Hortal \\
Department of Cardiac Surgery Anaesthesiology and \\
Resuscitation \\
c/ Dr Esquerdo, 46 \\
28007 Madrid \\
Spain \\
Dr José Anastasio Montero \\
Department of Cardiovascular Surgery \\
La Fe University Hospital \\
46009 Valencia \\
Spain
\end{tabular}

Dr Angel Candela-Toha

Department of Cardiac Surgery Anaesthesiology and Resuscitation

Hospital Ramón y Cajal

Carretera Colmenar Viejo km, 9, 100

28034 Madrid

Spain

Dr Jerrold Levy

Emory University Hospital

1364 Clifton Road Northeast

Atlanta, GA 30322

Dr Nanette Schwann

Lehigh Valley Health Network

1245 S Cedar Crest Blvd, Suite 301,

Allentown, PA 18103

Dr Frank Sellke

Rhode Island Hospital

593 Eddy St/APC 425

Providence, RI 02903

Dr Howard Song

Division of Cardiothoracic Surgery

Oregon Health and Science University

3181 SW Sam Jackson Park Road, L 353

Portland, OR 97239

Dr Luis Velez-Pestana

The Methodist Hospital

6565 Fannin B452

Houston, TX 77030
Dr Takeshi Shimamoto

\section{Dr Chikara Ueki}

Dr Keisuke Watadani

Dr Shun Watanabe

Dr Mónica Barranco

Dr Jose Maria Barrio

Dr Alejandro Garrido

Dr Mario Iglesias

Dr Mario Begoña Quintana

Dr Guillermo Rodriguez

Dr Eduardo Sanchez

Dr Ana Bel Minguez

Dr Lucia Doñate

Dr Tomás Heredia

Dr Juan Antonio Margarit

Dr Daniel Mata

Dr Azucena Pajares

Dr Manuel Pérez

Dr Francisco José Valera

Dr Rosario Vicente

Dr Salvador Torregrosa

Dr Ignacio Garcia Andrade

Dr José Garrido

Dr Marcos Martínez

Dr Adolfo Martínez Pérez

Dr Enrique Oliva

Dr Diego Parise

Dr Marc Azran

Dr Roman Sniecinski

Dr Gautam Sreeram

Dr William Whitley

Dr James Wu

Dr Nikola Dobrilovic

Dr William Feng

Dr James Fingleton

Dr Victoria Miller

Dr Arun Singh

Dr Lynn Boshkov

Dr Matthew Slater

Dr Steven Guyton

Dr Lauren Brunner

Dr Deanna Cully

Dr Frederick Tibayan

Dr Paul Wacek

Dr Brian Bruckner

6

Dr Nidal Abdel-Rahman

Dr Nicolas Athanassiou

Dr Ghazala Butt

Dr James Carter

Dr Diane Gibson

Dr Elizabeth Herrera 


\begin{tabular}{ll}
\hline & Dr Jessica Brown \\
& Dr Joseph Naples \\
& Dr Michael Reardon \\
& Dr Hany Samir \\
& Dr Karanbir Singh \\
& Dr Zbigniew Wojciechowski \\
Dr Linda Sundt & Dr James Diehl \\
Department of Anaesthesiology & Dr Elia Elia \\
Thomas Jefferson University & Dr Rehana Jan \\
111 South 11th St, Suite G8490 & Dr Scott Silvestry \\
Philadelphia, PA 19107 & \\
Dr Ian Welsby & Dr Joseph Mathew \\
Department of Anaesthesiology, & \\
Duke University Medical Center, & \\
2301 Erwin Rd, 5691-H HAFS Bldg & \\
Durham, NC 27710 & \\
\hline
\end{tabular}

\section{APPENDIX 2. Data monitoring committee}

Mark L. Barr, MD (Chair); Mark S. Slaughter, MD; Michael C. J. Wanscher, MD; Marc Buyse, Biostatistician. 